\title{
Societal Threat and Cultural Variation in the Strength of Social Norms: An Evolutionary Basis
}

\author{
Patrick Roos', Michele Gelfand², Dana Nau', \& Janetta Lun². \\ ${ }^{1}$ Department of Computer Science, ${ }^{2}$ Department of Psychology \\ University of Maryland, College Park
}

Acknowledgements: This research was based on work supported in part by the U.S. Army Research Laboratory and the U.S. Army Research Office under Grants W911NF-08-1-0144 and W911NF-11-1-0344, and the U.S. Airforce Grants FA9550-12-1-0021 and FA955-01-41-0020. 


\begin{abstract}
The strengths of social norms vary considerably across cultures, yet little research has shown whether such differences have an evolutionary basis. Integrating research in crosscultural psychology with evolutionary game theory, we show that groups that face a high degree of threat develop stronger norms for organizing social interaction, with a higher degree of normadherence and higher punishment for deviant behavior. Conversely, groups that have little threat can afford to have weaker norms with less punishment for deviance. Our results apply to two kinds of norms: norms of cooperation, in which individuals must choose whether to cooperate (thereby benefitting everyone) or enrich themselves at the expense of others; and norms of coordination, in which there are several equally good ways for individuals to coordinate their actions, but individuals need to agree on which way to coordinate. This is the first work to show that different degrees of norm strength are evolutionarily adaptive to societal threat. Evolutionary game theoretic models of cultural adaptation may prove fruitful for exploring the causes of many other cultural differences that may be adaptive to particular ecological and historical contexts.
\end{abstract}

Keywords:

Punishment, Norms, Cultural Evolution, Evolutionary Game Theory, Public Goods Game, Coordination 


\section{Societal Threat and Cultural Variation in Strength of Social Norms:}

\section{An Evolutionary Basis}

The development and enforcement of social norms is a unique feature of human sociality that transcends history and groups. The capacity for social learning and cultural transmission enables humans not only to develop, maintain, and enforce social norms, but also to pass them on to future generations. For any cultural group, social norms serve a critical function in that they enable the group's members to coordinate social action and accomplish tasks. Yet while social norms are universal, the strength of social norms varies widely around the globe. Research has shown, for example, considerable cross-cultural variation in norms for fairness, cooperation, and the willingness to punish to enforce such norms (Balliet \& Van Lange, 2013; Ensminger \& Henrich 2014; Gelfand, Raver, Nishii, Leslie, Lun et al., 2011; Henrich et al. 2006, 2010, 2012; Herrmann, Thöni, \& Gächter, 2008). Understanding the evolution of norms and how differences in norm strength arise through the process of cultural adaptation is an important part of understanding our complex social world.

In this research, we explore how cultural differences in norm strength, defined as degree of adherence to norms and punishment of norm-deviance (Gelfand et al., 2011), emerge from the evolutionary process of cultural adaptation. While there are many different types of social norms, we focus on norms for organizing social interaction, which includes both cooperation and coordination norms. We test the notion that cultures' exposure to societal threat is a mediating factor in differences in norm strength. In contexts of high threat-whether it is ecological threats like natural disasters - or manmade threats such as threats of invasions - we expect societies evolve to have stronger norms for coordinating social interaction because they are necessary for 
survival. By contrast, in contexts low threat, we expect there to be less need to coordinate social action, affording weaker norms and more tolerance for norm violating behavior.

Some indirect evidence for this supposition can be found across numerous disciplines. For example, political scientists have long argued that when nations are involved in external conflicts and must face the possibility (or reality) of invasion from foreign nations, they need to develop internal order and cohesion in order to successfully deal with the enemy (Cosner, 1956; Sumner, 1906). As cogently argued by (1906), "the exigencies of war with outsiders are what make peace inside, lest internal discord should weaken the we-group for war" (p. 12) (see also Kesebir, 2012). Likewise, anthropological research has shown that groups that have a dearth of natural resources need strong norms for coordination for survival (Lomax and Berkowitz, 1972). More recently, Gelfand et al. (2011) found that societies that have had high degrees of territorial threats, low natural resources (e.g., food supply), and high degrees of natural disasters (e.g., floods, cyclones, and droughts) were tight, i.e., had more compliance with social norms and a restriction of range of what was appropriate, as compared to societies that were loose and had low levels of these threats. Nevertheless, while such studies provide evidence that threat may be an important factor in the evolution of strong norms for organizing social interaction, no research to date has examined whether groups actually require stronger norms and associated punishment of deviance in order to survive under high threat. More generally, whether differences in punishment across societies have any evolutionary basis remains unclear.

To fill this void, we use evolutionary game theoretic (EGT) modeling to explore whether stronger norms with higher punishment of deviance are evolutionarily adaptive under conditions of higher societal threat. Norms are a very broad concept, with many types of norms that differ in various ways - but as noted above, we are specifically interested in social norms that facilitate organized social action. Within this subset of norms, we examine two general types of norms: 
norms of cooperation and norms of coordination. In norms of cooperation, an individual has a temptation to not adhere to the norm of cooperating in order to acquire an immediate benefit at the other's expense. In norms of coordination there exists no such temptation, only different ways of coordinating. To explore these norms we use cooperation games (Study 1) and coordination games (Study 2), and in both studies we assess how threat affects the strength of norms, including the degree to which deviance from the norm is tolerated or punished, that emerges in populations.

Before we describe our model and results in detail, we first provide a brief primer on relevant concepts and techniques from evolutionary game theoretic modeling. Evolutionary Game Theory Applied to Human Behavior and Cultural Adaptation

The evolutionary game theoretic (EGT) computational models that we employ in this research are best viewed as a complementary methodology to other methods appearing in this special issue. Computational modeling, including EGT and computational multi-agent system models, are increasingly being used in psychological science. As a complementary approach to study social and organizational phenomena, organizational scholars have described computational modeling as the "third scientific discipline" (Ilgen \& Hulin, 2000). Computational approaches have been fruitfully applied to topics such as motivation (Scherbaum \& Vancouver, 2010; Vancouver, Weinhardt, \& Schmidt, 2010b; Vancouver, Putka, \& Scherbaum, 2005), job attitudes and withdrawal (Seitz, Hulin, \& Hanisch, 2000), personality (Read \& Miller, 2002), gender (Martell, Lane, \& Emrich, 1996), among others (Ilgen \& Hulin, 2000). Tutorials and primers on computational approaches have appeared in journals such as Journal of Applied Psychology (Vancouver et al. 2010b), Journal of Management (Vancouver, Tamanini, \& Yoder, 2010a), and Organizational Research Methods (Vancouver et al., 2005). We build on this effort 
and use EGT modeling to gain insights into the dynamics of social norms for organizing social interaction and the enforcement of such norms through punishment.

Evolutionary game theory (Alexander, 2009; Hofbauer \& Sigmund, 1998; Smith, 1980;

Weibull, 1997) studies the effects of (socio-cultural or biological) evolutionary pressures on populations of agents under the general framework shown in Figure 1. It assumes a population of agents with assigned strategies (i.e. behaviors) at time $t$. These agents interact in a game that models a situation of interest, e.g. a prisoner's dilemma game (Rapoport, 1965). After agents interact in the game and are assigned payoffs based on the game's definition, they reproduce into the next population at time $t+1$, according to a reproduction rule in which the agents' reproductive fitness depends on their payoffs. Generally speaking, agents who received a high payoff at time $t$ are "more fit" in the sense that their strategies are likely to be used by a larger number of agents at time $t+1$. In the context of human social behaviors, agents' strategies represent behaviors; and reproduction is not necessarily biological, but may be interpreted as the processes of learning (Harley, 1981) or the cultural transmission and change of memes, behaviors, and norms in human societies (Boyd \& Richerson, 1988; Dawkins 1976). As such, reproduction of behaviors reflects humans' unique capacity for social learning (Schlag, 1998; 1999; Taylor \& Jonker, 1979; Traulsen \& Hauert, 2009).

Since human social systems are highly complex, EGT models are highly simplified models that omit most of the details of human interactions. The aim is to design models so that they accurately reflect the essential nature of the interactions that are being studied. Such models are too abstract to provide exact numeric predictions of human behavior, but these models can be used to provide explanations of the central dynamics underlying the interactions and behaviors of interest. The basic effects of various factors on evolutionary outcomes can be tested through 
“virtual experimentation” by computer simulation (Winsberg, 2003), and support for causal relationships between these factors and outcomes can be established. Since the evolution of behaviors through social learning and cultural adaptation in populations over time is difficult if not impossible to study in laboratory or field studies, EGT modeling provides a useful tool to apply and explore related theories and hypothesis.

The evolutionary games framework adds an important dynamical approach for studying how human behaviors in populations evolve over time. Often, aside from describing the evolutionary trajectories and interactions of different behaviors or strategies, the aim is to find and describe evolutionary stable states. An evolutionary stable state, informally speaking, is one in which the relative proportions of strategies in a population have stabilized, and the population will revert to these same proportions if one introduces an arbitrarily small number of new agents with different strategies (Smith, 1980). In terms of culture, a stable state in strategies represents the behavioral norms that are adaptive and can be expected to remain in a population under the given conditions.

To this date, EGT approaches have been used to study the evolution of a great variety of social and cultural phenomena. Examples of such phenomena studied through evolutionary games include cooperation, altruism, and reciprocity (e.g., Axelrod \& Dion, 1988; Axelrod \& Hamilton, 1981; Bendor \& Swistak, 1995; Bowles \& Gintis, 2004; Colman, 2003; Boyd \& Richerson, 2009; Darwne \& Yao, 2002; Henrich, 2004; Nowak, 2006; Nowak, Tarnita, \& Wilson, 2010; Nowak \& Sigmund, 1992; Ohtsuki \& Nowak, 2007; Riolo, Cohen, \& Axelrod, 2001; Santos, Santos, \& Pacheco, 2008; Sethi \& Somanathan, 2001; Traulsen \& Nowak, 2006; Traulsen, Semmann, Sommerfeld, Krambeck \& Milinski, 2010), trust and reputation (e.g., Brandt et al. 2003; Fang, Kimbrough, Pace, Valluri, \& Zheng, 2002; Hauert, 2010; Kimbrough, 
2005; Mui, Mohtashemi, \& Halberstadt, 2002), fairness and empathy (Binmore, 1998; Nowak, Page, \& Sigmund, 2000; Page \& Nowak, 2002), punishment (e.g., Boyd et al. 2003; Boyd \& Richerson, 1992; Brandt et al., 2003; 2006; Hauert, De Monte, Hofbauer \& Sigmund, 2002; Hauert, Traulsen, Brandt, Nowak, \& Sigmund, 2007; Henrich \& Boyd, 2001; Henrich et al., 2010; Rand \& Nowak, 2011; Roos, Gelfand, Nau, \& Carr, 2014; Sigmund, Hauert, \& Nowak, 2001; Sigmund, De Silva, Traulsen, \& Hauert, 2010; Ye, Tan, Ding, Jia, \& Chen, 2011), social learning (e.g., Schotter \& Sopher, 2003), and communication and evolution of opinions (e.g., Chiu \& Qiu, 2014). However, this approach has not heretofore been used to understand cultural differences in norm strength.

\section{Methods}

Our evolutionary game theoretic model is designed to understand the process of cultural adaptation of populations of agents in both cooperation games and coordination games. Agents interact in a game phase and then in a punishment phase. Agents receive payoffs from these interactions and reproduce according to fitness. As a measure of the norm strength in a population, we use the percentage of agents who adhere to the norm, and the proportion of normenforcers - agents that would punish others for deviating from the norm. Our results are comprised of the evolutionary outcomes of the process of cultural adaptation, including the evolutionary trajectories of behaviors and the composition of behaviors in populations that have arrived at a stable state. We determine these results through multi-agent computer simulations.

Since we are specifically interested how societal threat shapes the evolutionary pressures and outcomes in the cultural adaptation process, we explore our model under varying degrees of societal threat, implemented as follows. First, our model assumes agents all acquire a base payoff (base-pay) from the environment, independent of the game interactions that we model explicitly. 
This is an abstraction that serves to capture the fact that our game models just one of the many kinds of interactions that individuals might have in a more realistic world. Then, the level of societal threat is implemented by subtracting an amount $\tau$ from this base payoff. Since threats like drought, hurricanes, tornadoes, famine, or hostile invasions all can reasonably be assumed to reduce the general payoff that agents in a population receive from their environment, implementing threat in this manner captures the essential effect of a broad array of threats. For instance, ecological threats are related to the availability of natural resources in that they often diminish agricultural yields and engender food shortages (Popp, 2006), and managing them often requires the use of the population's resources.

After agents receive the base payoff and payoffs from their interactions, the total acquired payoff $p$ is transformed to reproductive fitness through a fitness function that captures the wellestablished principle of diminishing marginal utility, i.e. increased payoffs produce diminishing marginal increases in fitness. This diminishing marginal utility captures the concept that the fifth meal of a day is not as important to an agent's fitness or well being as the first. A fitness transformation with this property is supported by an abundance of evidence across disciplines that increased resources lead to diminishing increases in utility (Diener \& Biswas-Diener, 2002; Diener, Kahneman, Tov \& Arora, 2010; Foster, 2004; Frey \& Stutzer, 2010; Veenhoven, 1989), and has appeared in several other works that use evolutionary game theory to study social or biological evolution (Foster, 2004; Godfray, 1991; Grodzinski \& Johnstone, 2012).

The transformation of payoff $p$ into fitness that we use is given by $f(p)=1-e^{-0.1 \cdot p}$, which captures the notion that increased payoffs $(p)$ produce diminishing marginal increases in 
fitness ${ }^{1}$. Different levels of threat are implemented through a threat level that reduces the agents' base payoff by an amount $\tau$. Note the payoff $p$ that goes into the fitness function is the total payoff an agent has acquired, including the base payoff. So accounting for threat $\tau$, we have an agent's payoff $p=$ base-pay - $\tau+x$, where $x$ is the payoff an agent acquires from its game interactions that we model. Hence, the level of threat shifts an agent's reference point (the payoff it has before interactions) to the left or to the right on the fitness curve. At very high levels of threat, the reference point is shifted far to the left, and the fitness of agent is close to 0 no matter how much payoff they acquire from the game interactions modeled. In this case agents the death rate wipes out the population; analogous to a catastrophic event that kills off a population, we are left with an empty grid. At very low levels of threat, the reference point is shifted far to the right on the fitness curve, where marginal returns of payoffs are negligible since the curve flattens more and more the higher the payoffs get. In this case, differences in game-payoffs again have very little effect on reproductive fitness, and all agents are effectively in a state of neutral drift. Since such extreme levels of high or low threat render the evolutionary outcomes trivial, our

${ }^{1}$ While there has been some debate over the exact shape of functions that relate measures of well-being and wealth, e.g. Stevenson and Wolfers, (2013) argue for a linear-logarithmic rather than sigmoidal function, all such proposed functions are monotonically increasing and have the property of diminishing marginal returns in payoffs, which is what is important for our main results (see Discussion). We have verified experimentally that our results are qualitatively similar in the case of both linear-logarithmic and sigmoid fitness functions. For the interested reader, more details about this issue are provided in the Supplemental Material. 
results generally focus on threat levels of

$0 \leq \tau \leq 30$

Our model assumes a spatial square lattice of 50x50 sites with periodic boundary conditions and von-Neumann neighborhoods (i.e., each site has four neighboring sites). Like existing models (Hammond, 2006, Hartshorn, 2013), we start with an empty lattice and then the simulation proceeds with each time step consisting of the following sequence of events:1) Birth: An agent with a random strategy appears at a random empty site. 2) Base Payoff: Each existing agent receives the base payoff from the environment; we use a base payoff of 30 throughout our experiments. 3) Interaction Payoff: Each agent plays a number of games (4 in our experiments) with other agents, receiving payoffs according to the game definition. After each game interaction is complete, each agent gets the chance to punish each of its co-players, in which case payoffs are reduced by the punishment cost and fine accordingly. 4) Fitness: Each agent is assigned fitness according to the agent's accumulated payoff through the fitness function described above. 5) Reproduction: In random order, each agent is given a chance to reproduce with probability equal to its fitness. If an agent gets a chance to reproduce, it places an offspring in a randomly chosen empty site in its neighborhood, if one exists. The offspring has the same strategy of its parent. 6) Mutation: Each agent, with probability $\mu$, updates its strategy to a randomly chosen one. Mutation is analogous to an exploration rate with which agents explore the available strategy space (Traulsen, 2010). 7) Death: Each agent has a chance $d$ of dying. If an agent dies, it is removed from the lattice.

We ran computer simulations with the above model to test the hypothesis that exposure to threat is an important causal factor in the emergence of strong norms under the evolutionary pressures of cultural adaptation. If exposure to threat is indeed a cause of the emergence of 
strong norms, agents that adhere to the norm and enforce the norm through punishment should thrive evolutionarily under higher threat, while other agents that do not enforce the norm should not fare well evolutionarily. In contrast, under lower threat, we would expect the evolutionary pressures to allow for a greater variety of strategies and less norm-enforcing punishers.

We have applied this general evolutionary game model to several games of social interaction, including the Public Goods Game (PGG), Prisoner's Dilemma, and Stag Hunt. For reasons of space, our presentation focuses primarily on the PGG as an example of a cooperation norm (described in Study 1) and a simple coordination game as an example of a coordination norm (described in Study 2). Our results are robust and replicate to the other games, as shown in the supplementary material.

\section{Results}

\section{Study 1: Public Goods Game}

The PGG is a well-established paradigm for studying cooperation norms that has also been used to study punishment, i.e. norm enforcement (Brandt et al., 2003, 2006; Traulsen et al., 2010; Hauert, 2010, Hilbe \& Traulsen, 2012). Each participant in a PGG may either choose to Cooperate by contributing an amount $c$ to the public good, or Defect by contributing nothing. The sum of all contributions is multiplied by a factor $r$, capturing the benefit of cooperation. The resulting payoff pool is divided evenly among all players, regardless of whether they contributed. Since Defectors can get this payoff without the cost of contributing, the Nash equilibrium is for all players to defect. However, several studies have shown that punishment can foster and establish cooperative behavior as a societal norm (Boyd et al., 2003; Brandt et al., 2003, 2006; Hauert, 2002; Hauert, 2007; Hilbe \& Traulsen, 2012; Traulsen et al., 2010; Sigmund, et al., 2001; Roos et al., 2014; Ye et al., 2011). Our model of punishment is similar to these previous models: 
a player punishes by paying a cost $\lambda$ to reduce another player's payoff by an amount $\rho$. Since reputation for punishment is essential for cooperation, we allow agents to know the punishment strategy of their co-players. As a reference for the reader, Table 1 lists all of the model parameters with their default values and a short description.

An agent's full strategy is composed of a contribution strategy that determines its action to Cooperate or Defect in the cooperation game, and a punishment strategy that determines its decision of which actions to punish in the cooperation game. The strategies available to agents in our model are equivalent to those of an established state-of-the-art model of the evolution of punishment (Hilbe \& Traulsen, 2012, Roos et al., 2014). This model includes four punishment strategies: agents can punish (R)esponsibly (only punish Defectors, which we might call normenforcing agents), (A)ntisocially (only punish Cooperators), (S)pitefully (punish indiscriminately), or they can be (N)on-punishing (punish no-one). Since humans are known to sometimes punish antisocially (Henrich, McElreath, Barr, Ensminger, Barrett, Bolyanatz, et al., 2006) and the inclusion of antisocial punishment can influence evolutionary dynamics of cooperation and punishment in important ways (Rand, Armao, Nakamaru, \& Ohtsuki, 2010; Rand \& Nowak, 2011), allowing for the full set of these punishment strategies is important. Agents may take the punishment reputation of their neighbors into account when deciding on whether to cooperate or not. Hence there are three possible contribution strategies: agents can either always (C)ooperate, always (D)efect, or be $(\mathbf{O})$ pportunistic. Opportunistic agents take the punishment reputation of neighbors in account when deciding to cooperate or defect in the cooperation game phase. Given the punishment strategies of its neighbors, they Defect or Cooperate depending on whichever action gives them a higher expected payoff in the current game and punishment phase. This decision of an Opportunistic agent to contribute can be 
reduced to the condition that the number of Responsible Punishers in the PGG group minus the number of Anti-Social Punishers in the group is greater than $c / \rho$, as derived in the Appendix. With the parameters of our model, this simplifies to the rule: contribute if there are more Responsible Punishers in the group than Anti-Social Punishers. To aid reader's intuition of the interactions and outcomes, Table 2 gives some examples of interactions and outcomes when different combinations of strategies play a PGG with punishment; it focuses on groups with Opportunistic (O) agents since the Cooperative (C) and Defective (D) agents simply always contribute or defect respectively. The table assumes the same model parameters as used in our main experiments.

Given these strategies for the PGG, we ran simulations of the evolution of populations under varying degrees of threat (in increments of 5) and observed the evolutionary trajectories of strategies and the stable state strategy proportions which populations settle on after running the simulation for sufficient time. We used the long-term average proportions of strategies after running simulations for a large number of time-steps as an estimate of the strategy proportions in the evolutionarily stable state. As mentioned before, we measured norm strength as the percentage of actions that adhere to the norm and the extent to which deviance from the norm would be punished, measured as the proportion of norm-enforcing punishers in the population.

Figure 2 shows the long-term average proportions of the strategies in the population, as a function of a constant level of threat $\tau$ to which the population is exposed. The left panel shows the contribution strategies (in the game phase), while the right panel shows the different punishment strategies (in the punishment phase). The stable proportions shown represent the composition of behaviors in populations that the cultural evolutionary pressures lead to and that the population settles on through the process of cultural adaptation. We can see that the 
percentage of cooperative actions, also shown in the left panel, increases with increased threat, illustrating that cooperation becomes a norm in the population. Under high threat, the extra payoff achieved from cooperating has a large effect on agents' fitness, and hence strategies that flourish are those that cooperate. The right panel shows that norms also develop for punishing defection, i.e. deviations from the established cooperation norm, illustrating that responsible punishers proliferate under conditions of high threat. Under low threat, the extra payoff of cooperation has little effect on agent's fitness, and hence cooperators and responsible punishers proliferate less, i.e. evolutionary pressures toward the cooperation norm and punishment of deviance are weaker. Since the degree of norm adherence and norm enforcement are a measure of norm strength, these results show how increased threat result in the emergence of increased strength of norms within a population.

Figure 3 show snapshot of the population grid of our simulations in different stages under conditions of high threat. Each square represents an agent and the color represents the strategy that the agent uses. The left panel shows the cooperation strategies and the right panel shows the punishment strategies. In the right panel, one can observe clusters of Responsible (R) Punishers emerging and growing to take over the majority of the population. These Responsible Punishers maintain cooperation in the PGG by agents around them by punishing defectors. The Opportunistic (O) agents in the left panel largely cooperate along with the Cooperator (C) agents. Some Non-Punishers (N) remain, as long as the agents around them are cooperating. Spiteful (S) and Anti-Social (A) Punishers are rare. Video files in color of simulations under high and low threat are provided in the Supplementary Material.

The results in Figures 2 and 3 showed where evolutionary pressures lead to if the degree of threat remains constant over time. Threat levels however may change over time and 
established norm strength in a population change as a response to changes in threat. Figure 4 show single run results showing how a population adapts its norm strength in response to threats. Part a) shows how in response to a sudden increase in threat, a population's norm strength increases rapidly, and part b) shows that the norm strength decreases at a somewhat more gradual rate after the threat vanishes. Conceptually this is analogous to a situation where a group that has had very low threat suddenly experiences a large threat (ecological, e.g., natural disaster, or human threat, e.g., terrorism), or vice versa. One can observe the norm-adherence rate and proportion of norm-enforcers increasing rapidly as a result of an increase in threat, while a decrease in threat leads to a (more gradual) decline in the proportion of norm-enforcing punishers in the population.

The result that increased threat increases norm strength under cultural evolutionary pressures is robust to various other parameter changes and model choices. We have confirmed this by running an array of simulation experiments with different games and parameter variations in the multiplication factor $r$, the punishment fine $\rho$, the death rate $d$, the mutation rate $\mu$, and the degree to which punishment reputation is known. Also, since our populations are structured on a grid and it is known that population structure alone can aid cooperation under certain conditions, we have performed baseline experiments without punishment in order to untangle the effects of punishment from effects of population structure alone. Repeating our simulations with identical conditions, but without the punishment phase, shows that while spatial structure alone can provide for the evolution of some cooperation (approximately 50\%), punishment is necessary to achieve the high levels of cooperation under threat achieved by populations in Figure 2 . This is consistent with the results of previous work that introduced the spatial PGG with punishment and showed that punishment significantly aided the evolution of cooperation (Brandt et al., 2003). 
We have also tested the effects of counter-punishment (as in Hilbe \& Traulsen, 2011) and found that general relationship between threat and tightness holds even for the case of certain counterpunishment. Finally, since punishment reputation isn't necessarily always known, we have also tested our model when the punishment reputation of co-players is known with a certain probability only, and found that the results are also robust to this change. The results for all these experiments are provided in the Supplementary Material.

\section{Study 2: Coordination Game}

Coordination games are games in which the agents receive high payoffs only if they choose mutually consistent actions. For example, if two drivers approach each other on a road, the outcome is good for both if they both drive on the left or both drive on the right, and bad for both if one drives on the left and the other drives on the right. In a coordination game, these outcomes would be modeled by high payoffs and low payoffs, respectively. For the coordination games in this article, we focus on two-player games. Hence, instead of each agent playing a game with all of its neighbors as for the PGG, for the coordination game each agent plays a paired game each with four other agents. In each of these game interactions, both agents receive a chance to punish the other. Figure 5 shows the payoff matrix for the coordination game used in our experiments. We assume there are two norms, norm A and norm B, and they are equivalent in terms of the payoffs achieved by individuals if they coordinate on either norm. As before, agents play a game phase and a punishment phase, except that now the game phase is not a contribution phase but a coordination phase.

The strategies available to our agents are similar to the ones in Study 1, with some changes to adapt the strategies to the coordination game. In the coordination game phase agents can always play coordination action A (Norm A Adhering), always play coordination action B 
(Norm B Adhering), or again play (O)pportunistically, choosing A or B depending which is expected to give a better result based on the punishment reputation of co-players. Both A and B are potential norms if they are established as the common action to take in a population. In the punishment phase, agents can be a Norm A Enforcer (E-A) by punishing B players, be a Norm B Enforcer (E-B) by punishing A players, or, as before, punish (S)pitefully (punish indiscriminately), or be (N)on-punishing (punish no-one). Opportunistic agents in this case play $\mathbf{A}$ if their partner is an E-A agent, $\mathbf{B}$ if their partner is an E-B agent, and randomly otherwise.

We ran the same kinds of simulation experiments for the coordination game as we did for the PGG in Study 1, observing the evolutionary trajectories of strategies and the stable state strategy proportions which populations settle on under varying degrees of societal threat. Figure 6 shows the long-term average proportions of the punishment strategies in the population, as a function of the level of threat $\tau$ that the population is exposed to. As in Figure 2, the stable proportions here represent the composition of behaviors in populations that the cultural evolutionary pressures lead to and that the population settles on through the process of cultural adaptation. Since the two possible norms A and B are equivalent in terms of payoff, each given simulation may settle on a different norm (A or B) by chance, with either mostly Norm A Enforcers (E-A) or Norm B Enforcers (E-B) respectively. Since we are averaging over many simulations and are interested in the strength of norms that evolves (regardless of whether it is A or B that emerges as the norm), the plot reports only the punishment strategies and therein the long term proportions of E-A and E-B agents are aggregated into one proportion capturing all Norm-(E)nforcing agents (labeled E). The panel also shows the percent of interactions in which both agents successfully achieved coordination (\% coordination). The qualitative results are identical to the results of Study 1 . While in Study 1 the percentage of actions that were 
cooperative increased with threat, here the percentage of coordination increases with increased threat. Similarly, the stable proportion of Norm-(E)nforcing punishers is low when $\tau$ is low, and high when $\tau$ is high. Since the degree of norm adherence and norm enforcement are a measure of norm strength, these results again show how increased threat result in the emergence of increased strength of norms within a population.

\section{Summary}

Our results have shown how, both in games of cooperation (Study 1) and coordination (Study 2), increased threat leads to the emergence of stronger norms for organizing social interaction and higher degrees of punishment of norm-deviant behavior. In order to explain these results, and the mechanisms that lead to them, it is important to understand that the shape of the fitness function plays a crucial mediating role. Under conditions of high threat, agents operate in the space of lower payoffs, where fitness increases more quickly with a given increase in payoff than under low threat. This increases the selective pressure for coordinated or cooperative interactions, and leads to the adoption of behaviors that adhere to and enforce a norm. See Figure 7, which shows the expected payoff and corresponding fitness a given agent receives when it plays four games with others that play the established norm (are coordinating) vs. when all coplayers are playing a random action in the coordination game under both a high threat $(\tau=32)$ and low threat $(\tau=10)$ condition. Under high threat the payoff acquired from coordinating on a norm has a large positive effect on agents' fitness, while under low threat it has a relatively small effect. It is this difference that makes coordinating agents under high threat have a significant evolutionary advantage over agents that are coordinating less, and hence strong coordination norms with punishers of agents who do not conform to the norm evolve and take over the population. The same logic applies to norms of cooperation. 


\section{Discussion}

Strength of social norms and associated norm-enforcement differ widely around the globe. The models and experiments presented in this paper demonstrate that high levels of threat create evolutionary pressures toward stronger norms for organizing social action, whereas low levels of threat allow for cultural populations with weaker norms. Cultural groups facing chronic high levels of threat evolve higher norm-adherence and higher levels of norm-enforcement than cultures exposed to relatively low levels of threat, both in cooperation as well as coordination games, and these results are mediated by the property of diminishing marginal returns in fitness of payoffs. Our models also show that temporary increases in threat cause norm strength to increase until the threat subsides, at which point behavioral norms begin returning to their previous level. This is analogous, for example, to situations where a society that has had relatively low levels of threat is faced with a temporary threat on its own soil (e.g., the 9/11 in the U.S.). Thus these results support correlational research that show that exposure to threat is an important driving factor in the evolution and maintenance of cultural differences in normstrength and punishment of deviation (Gelfand et al, 2011; Harrington \& Gelfand, 2014).

This research makes a number of novel contributions. Previous evolutionary models of punishment have focused on whether the existence of punishment is viable under cultural evolutionary pressures and how it can aid the evolution of cooperation (Brandt et al., 2003, 2006; Hauert et al., 2002; Hauert et al., 2007; Henrich \& Boyd, 2001; Hilbe \& Traulsen, 2012; Jaffe \& Zaballa, 2010; Traulsen et al., 2009; Ye et al., 2011). Our research extends this work to into new directions by accounting for the evolution of cultural differences in punishment of normdeviance. Furthermore, while previous evolutionary models have shown how between-group 
warfare can promote within-group cooperative behavior (e.g., Bowles 2009; Nowak, 2006), our model is designed to capture a broad array of different types of threat and shows evolution toward strong cooperation or coordination norms without the requirement for group selection (Bowles, 2006; Henrich, 2004; Traulsen \& Nowak, 2006). That is, our model shows that differences in norm strength can emerge independently of evolutionary pressures that derive from competition with other groups.

This research also contributes to cross-cultural psychology by demonstrating the value of evolutionary game theoretic modeling for testing the notion that cultural values and norms develop as adaptations to their ecology. To date, this has been an implicit assumption in ecological models of culture (Berry \& Annis, 1974; Berry 1975; Cohen \& Nisbett, 1994; Nisbett, 2004; Segall et al., 1966; Triandis, 1972; Whiting \& Whitting, 1975), which have a long tradition in the field. For example, in their research on the strength of social norms, Gelfand et al., (2011) argued that tight societies — which have strong norms and little tolerance for deviance - require stronger norms as adaptations to ecological and historical threats that these societies experience on a chronic basis. Historians and geographers have also joined anthropologists and psychologists in advancing this perspective, as evident in the widely acclaimed Guns, Germs, and Steel, a treatise on the evolution of cultural differences (Diamond, 1998). Yet, despite the intuitively appealing nature of the widespread assumption that culture is an adaptation to ecological factors, it has yet to be fully tested. While correlational and experimental studies of ecology and culture can provide some indirect evidence to address this contention, they cannot address whether groups and the individuals comprising them develop different cultural adaptions because they are required for survival or evolutionarily adaptive under different ecological 
conditions. Our model has shown precisely that this is the case for societal threat and the development of strong norms for organizing social action.

This research opens up a number of interesting avenues for future research. For example, although our theorizing and research focused on societal cultures, we expect it to also apply to the strength of norms in organizational contexts. It has long been argued that organizations are open systems that are affected by the external environments in which they are embedded (Emery \& Trist, 1965; Katz \& Kahn, 1978). As well, research has found that organizational cultures differ on the extent to which they have clear and agreed-upon rules for social interaction, with some organizations having high culture strength and others having weaker norms for coordination and more flexibility and experimentation (Cooke \& Rousseau, 1988; Cooke \& Szumal, 1993; Litwin \& Stringer, 1968; O’Reilly, Chatman, \& Caldwell, 1991; O’Reilly \& Chatman, 1996; Quinn, 1988; Rousseau, 1990). Our model would suggest that organizations that deal with conditions of great threat, danger, and vulnerability (e.g., the army, nuclear power plants) will evolve to have stronger organizational cultures as compared to those that have low threat (Gelfand, Nishii, \& Raver, 2006). As McKelvey (1982) cogently argued, "Environmental threat more than anything else seems to be accompanied by organizations having tight and extensive control systems" (p. 186). This also has implications for the types of leaders and employees that are adaptive in such circumstances, which would be an interesting area for further evolutionary game theoretic work.

The model can be expanded to explore additional factors that exist in the real world. For example, recent research has demonstrated the importance of institutional punishment as an aspect of the evolution of cooperation (Jaffe \& Zaballa, 2010; Sigmund, et al., 2010). Since institutional punishment acts as a replacement for individual punishment, strong (highly funded) 
institutions result in a decreased need for peer-punishment while weak (sparsely funded) institutions result in an increased need for peer-punishment. Most relevant to the current research is that societal threats are likely to weaken institutions by leading to a decrease in the overall payoff a society has and hence a decrease in the relative size of the institutional punishment funds available. With a decrease in funding for (or effectiveness of) punishing institutions due to societal threats, there is again a greater need for individual-based punishment to maintain the same degree of cooperation. Therefore the existence of institutional punishment mechanisms in a population would likely not change the general relationship between societal threat and punishment propensity illustrated in this paper. Other limitations of our model provide avenues for future work. In more complex models, agents might be bestowed with mental faculties to model uncertainty in threat, the ability to be mobile, to choose and prefer interaction partners based on certain criteria, to reward rather than punish (Wang \& Leung, 2010; Wang, Leung, \& See, 2011), and to have mixed strategies, among other factors.

\section{Conclusion}

As this special issue attests, research has made great strides in understanding differences in cultural norms. Our research expands upon this tradition through the use of EGT modeling to study the evolution of differences in norm strength. Our results support the theory that exposure to higher levels of societal threat leads to cultural adaptation of stronger norms for organizing social interaction and higher punishment of deviations from those norms. Explicit models of cultural adaptation akin to those presented in this paper may prove fruitful for exploring and understanding the causes of other cultural differences that may be adaptive to particular ecological and historical contexts. 


\section{References}

Alexander, J. M. (2009). Evolutionary Game Theory. The Stanford Encyclopedia of Philosophy. N. Z. Edward (Ed.). Retrieved from http://plato.stanford.edu/archives/fall2009/entries/gameevolutionary/

Axelrod, R., \& Dion, D. (1988). The further evolution of cooperation. Science, 242, 1385-1390.

Axelrod, R., \& Hamilton, W. D. (1981). The evolution of cooperation. Science, 211, 1390-1396.

Balliet, D., \& Van Lange, P. A. (2013). Trust, punishment, and cooperation across 18 Societies: A meta-analysis. Perspectives on Psychological Science, 8, 363-379.

Bendor, J., \& Swistak, P. (1995). Types of evolutionary stability and the problem of cooperation. Proceedings of the National Academy of Sciences, 92,,3596-3600.

Berry, J.W. (1975). An ecological approach to cross-cultural psychology. Nederlands Tijdschrift voor de Psychologie, 30, 51-84.

Berry, J. W., \& Annis, R. C. (1974). Acculturative stress: The role of ecology, culture and differentiation. Journal of Cross-Cultural Psychology, 5, 382-406.

Binmore, K. G. (1998). The evolution of fairness norms. Rationality and Society, 10, 275-301.

Bowles, S. (2006). Group competition, reproductive leveling, and the evolution of human altruism. Science, 314, 1569-72.

Bowles, S. (2009). Did warfare among ancestral hunter-gatherers affect the evolution of human social behaviors? Science, 324, 1293-8.

Bowles, S., \& Gintis, H. (2004). The evolution of strong reciprocity: cooperation in heterogeneous populations. Theoretical population biology, 65, 17-28. 
Boyd, R. \& Richerson, P. J. (1988). Culture and the evolutionary process. Chicago: University of Chicago Press.

Boyd, R. \& Richerson, P. J. (1992). Punishment allows the evolution of cooperation (or anything else) in sizable groups. Ethology and Sociobiology, 13, 171-195.

Boyd, R., Gintis, H., Bowles, S., \& Richerson, P. J. (2003). The evolution of altruistic punishment. Proceedings of the National Academy of Sciences, 100, 3531-3535.

Boyd, R., \& Richerson, P. J. (2009). Culture and the evolution of human cooperation. Philosophical Transactions of the Royal Society B: Biological Sciences, 364, 3281-3288.

Brandt, H.; Hauert, C.; \& Sigmund, K. (2003). Punishment and reputation in spatial public goods games. Philosophical Transactions of the Royal Society B: Biological Sciences, 270, 1099104.

Brandt, H.; Hauert, C.; \& Sigmund, K. (2006). Punishing and abstaining for public goods. Proceedings of the National Academy of Sciences, 103, 495-7.

Chiu, C-y., \& Qiu, L. (2014). Communication and culture: A complexity theory approach. Asian Journal of Social Psychology, 17, 108-111.

Colman, A. M. (2003). Cooperation, psychological game theory, and limitations of rationality in social interaction. Behavioral and brain sciences, 26, 139-153.

Cohen, D., \& Nisbett, R. E. (1994). Self-protection and the culture of honor: Explaining southern violence. Personality and Social Psychology Bulletin, 20, 551-567.

Cooke, R. A., \& Rousseau, D. M. (1988). Behavioral norms and expectations: A quantitative approach to the assessment of organizational culture. Group \& Organization Management, $13,245-273$. 
Cooke, R. A., \& Szumal, J. L. (1993). Measuring normative beliefs and shared behavioral expectations in organizations: The reliability and validity of the organizational culture inventory. Psychological Reports, 72, $1299-1330$.

Cosner, L., (1956). The functions of social conflict. New York: Free Press.

Darwne, P. J. \& Yao, X. (2002). Co-evolutions in iterated prisoner's dilemma with intermediatel levels of cooperation: Application to missile defense. International Journal of Computational Intelligence and Applications, 2, 83-107.

Dawkins, R. (2006). The selfish gene. Oxford, UK: Oxford university press.

Diener, E., Kahneman, D., Tov, W., \& Arora, R. (2010). Income's association with judgments of life versus feelings. In E. Diener, D. Kahneman, \& J. F., Helliwell (Eds.), International differences in well-being (pp. 3-15). Oxford, UK: Oxford University Press.

Ensminger, J., \& Henrich, J. (Eds.). (2014). Experimenting with Social Norms: Fairness and Punishment in Cross-cultural Perspective. New York: Russell Sage Foundation.

Emery, F. E., \& Trist, E. L. (1965). The causal texture of organizational environments. Human Relations, 18, 21-32.

Fang, C., Kimbrough, S. O., Pace, S., Valluri, A., \& Zheng, Z. (2002). On adaptive emergence of trust behavior in the game of stag hunt. Group Decision and Negotiation, 11, 449-467.

Foster, K. R. (2004). Diminishing returns in social evolution: the not-so-tragic commons. Journal of evolutionary biology, 17, 1058-1072.

Frey, B. S. \& Stutzer, A. (2010). Happiness and economics: How the economy and institutions affect human well-being. Princeton University Press.

Godfray, H. C. J. (1991). Signaling of need by offspring to their parents. Nature, 352, 328-330.

Gelfand, M. J., Nishii, L., \& Raver, J. (2006). On the nature and importance of cultural tightnesslooseness. Journal of Applied Psychology, 91, 1225-1244. 
Gelfand, M., Raver, J., Nishii, L., Leslie, L., Lun, J, et al. (2011). Differences between tight and loose cultures: A 33-nation study. Science, 332,, 1100-1104.

Grodzinski, U. \& Johnstone, R. A. (2012). Parents and offspring in an evolutionary game: the effect of supply on demand when costs of care vary. Proceedings of the Royal Society B: Biological Sciences, 279, 109-115.

Harbeck, M., Seifert, L., Hänsch, S., Wagner, D. M., Birdsell, D., Parise, K. L., ... \& Scholz, H. C. (2013). Yersinia pestis DNA from skeletal remains from the 6th century AD reveals insights into Justinianic Plague. PLoS pathogens, 9(5), e1003349.

Harley, C. B. (1981). Learning the evolutionarily stable strategy. Journal of Theoretical Biology, $89,611-633$.

Harrington, J., \& Gelfand, M. J. (2014). Tightness-looseness across the 50 United States. Proceedings of the National Academy of Sciences, 22, 7990-7995.

Hauert, C. (2010). Replicator dynamics of reward \& reputation in public goods games. Journal of Theoretical Biology, 267, 22-28.

Hauert, C., De Monte, S., Hofbauer, J., \& Sigmund, K. (2002). Replicator dynamics for optional public good games. Brain, 218, 187-194.

Hauert, C., Traulsen, A., Brandt, H., Nowak, M., \& Sigmund, K. (2007). Via freedom to coercion: the emergence of costly punishment. Science, 316, 1905.

Henrich, J. (2004). Cultural group selection, coevolutionary processes and large-scale cooperation. Journal of Economic Behavior \& Organization, 53, 3-35.

Henrich, J. \& Boyd, R. (2001). Why people punish defectors. Weak conformist transmission can stabilize costly enforcement of norms in cooperative dilemmas. Journal of Theoretical Biology, 208, 79-89. 
Henrich, J., Ensminger, J., McElreath, R., Barr, A., Barrett, C., Bolyanatz, A., ... \& Ziker, J. (2010). Markets, religion, community size, and the evolution of fairness and punishment. science, 327(5972), 1480-1484.

Henrich, J., McElreath, R., Barr, A., Ensminger, J., Barrett, C., et al. (2006). Costly punishment across human societies. Science, 312, 1767-70.

Herrmann, B., Thöni, C., \& Gächter, S. (2008). Antisocial punishment across societies. Science, 319, 1362-1367.

Hilbe, C., \& Traulsen, A. (2012). Emergence of responsible sanctions without second order free riders, antisocial punishment or spite. Scientific reports, 2.

Hofbauer, J. \& Sigmund, K. (1998). Evolutionary games and population dynamics. Cambridge, UK: Cambridge University Press.

Ilgen, D.R. \& Hulin, C. L. (2000) Computational modeling of behavior in organizations: The third scientific discipline. Washington DC: American Psychological Association.

Katz, D. \& Kahn, R. L. (1978). The social psychology of organizations (2nd Ed.). New York: Wiley.

Kesebir, S. (2012). The superorganism account of human sociality: How and when human groups are like beehives. Personality and Social Psychology Review, 16, 233-261.

Litwin, G. H., \& Stringer, R. A. (1968). Motivation and organizational climate. Boston: Harvard Business School Press.

Lomax, A. \& Berkowitz, N. (1972). The evolutionary taxonomy of culture. Science, 177, 228-39. Marlowe, F. W., Berbesque, J. C., Barr, A., Barrett, C., Bolyanatz, A., Cardenas, J. C., ... \& Tracer, D. (2008). More 'altruistic' punishment in larger societies. Proceedings of the Royal Society B: Biological Sciences, 275, 587-592. 
Martell, R.F., Lane, D.M., \& Emrich, C. (1996) Male-female differences: A computer simulation. American Psychologist, 51, 157-158.

McKelvey, B. (1982). Organizational systematics--taxonomy, evolution, classification. Los Angeles: University of California Press.

Mui, L., Mohtashemi, M., \& Halberstadt, A. (2002, July). Notions of reputation in multi-agents systems: a review. In Proceedings of the first international joint conference on Autonomous agents and multiagent systems: part 1 (pp. 280-287). ACM.

Nisbett, R. (2004). The geography of thought: How Asians and Westerners think differently... and why. New York: Simon and Schuster.

Nowak, M. A. (2006). Five rules for the evolution of cooperation. Science, 314, 1560-1563.

Nowak, M. A. \& Sigmund, K. (1992). Tit for tat in heterogeneous populations. Nature, 355, 250253.

Nowak, M. A., Page, K. M., \& Sigmund, K. (2000). Fairness versus reason in the ultimatum game. Science, 289, 1773-1775.

Nowak, M. A., Tarnita, C. E., \& Wilson, E. O. (2010). The evolution of eusociality. Nature, 466, 1057-1062.

Ohtsuki, H. \& Nowak, M. A. (2007). Direct reciprocity on graphs. Journal of Theoretical Biology, 247(3), 462-470.

O’Reilly, C. A. \& Chatman, J. A. (1996). Culture as social control: Corporations, cults, and commitment. Research in Organizational Behavior, 18, 157-200.

O'Reilly, C. A., Chatman, J., \& Caldwell, D. F. (1991). People and organizational culture: A profile comparison approach to assessing person-organization fit. Academy of Management Journal, 34, 487-516. 
Popp, A. (2006). The effects of natural disasters on long run growth. Major Themes in Economics, 1, 61-81.

Quinn, R. E. (1988). Beyond rational management: Mastering the paradoxes and competing demands of high performance. Jossey-Bass.

Rand, D. G., \& Nowak, M. A. (2011). The evolution of antisocial punishment in optional public goods games. Nature Communications, 2, 434.

Rand, D. G., Armao IV, J. J., Nakamaru, M., \& Ohtsuki, H. (2010). Anti-social punishment can prevent the co-evolution of punishment and cooperation. Journal of Theoretical Biology, 265, 624-632.

Rapoport, A. (1965). Prisoner's dilemma: A study in conflict and cooperation. University of Michigan Press.

Read, S. J., \& Miller, L. C. (2002). Virtual personalities: A neural network model of personality. Personality and Social Psychology Review, 6, 357-369.

Riolo, R. L., Cohen, M. D., \& Axelrod, R. (2001). Evolution of cooperation without reciprocity. Nature, 414, , 441-443.

Roos, P., Gelfand, M., Nau, D., \& Carr, R. (2014). High strength-of-ties and low mobility enable the evolution of third-party punishment. Proceedings of the Royal Society B: Biological Sciences, 281(1776), 20132661.

Rousseau, D. M. (1990). Assessing organizational culture: The case for multiple methods. In B. Schneider (Ed.), Organizational climate and culture (pp. 153-192). San Francisco: JosseyBass.

Santos, F. C., Santos, M. D., \& Pacheco, J. M. (2008). Social diversity promotes the emergence of cooperation in public goods games. Nature, 454, 213-216. 
Scherbaum, C. A., \& Vancouver, J. B. (2010). If we produce discrepancies, then how? Testing a computational process model of positive goal revision. Journal of Applied Social Psychology, $40,2201-2231$.

Schotter, A., \& Sopher, B. (2003). Social learning and coordination conventions in intergenerational games: An experimental study. Journal of Political Economy, 111, 498-529.

Seitz, S. T., Hulin, C. L., \& Hanisch, K. A. (2000). Simulating withdrawal behaviors in work organizations: An example of a virtual society. Nonlinear Dynamics, Psychology, and Life Sciences, 4, 33-65.

Sethi, R., \& Somanathan, E. (2001). Preference evolution and reciprocity. Journal of Economic Theory, 97,,273-297.

Sigmund, K.; De Silva, H.; Traulsen, A.; \& Hauert, C. (2010). Social learning promotes institutions for governing the commons. Nature, 466, 861-863.

Sigmund, K., Hauert, C., \& Nowak, M. A. (2001). Reward and punishment. Proceedings of the National Academy of Sciences, 98, 10757-10762.

Smith, J. M. (1982). Evolution and the Theory of Games. Cambridge university press.

Smith, J.M. (1980). Evolutionary game theory. In C. Barigozzi, (Ed.) Vito Volterra symposium on mathematical models in biology (pp. 73-81). Springer

Stevenson, B., \& Wolfers, J. (2013). Subjective well-being and income: Is there any evidence of satiation? (No. w18992). National Bureau of Economic Research.

Sumner, W. G. (1906). Folkways: A study of the sociological importance of usages, manners, customs, mores, and morals. Boston: Ginn and Co.

Taylor, P. D. \& Jonker, L. B. (1978). Evolutionary stable strategies and game dynamics. Mathematical Biosciences 40, 145 - 156. 
Traulsen, A. \& Hauert, C. (2009). Stochastic evolutionary game dynamics. Reviews of Nonlinear Dynamics and Complexity, 2, 25-61.

Traulsen, A., Hauert, C., De Silva, H., Nowak, M. A., \& Sigmund, K. (2009). Exploration dynamics in evolutionary games. Proceedings of the National Academy of Sciences, 106, $709-712$.

Traulsen, A., Nowak, M. A., \& Pacheco, J. M. (2006). Stochastic dynamics of invasion and fixation. Physical Review E, 74(1), 011909.

Traulsen, A. \& Nowak, M. A. (2006). Evolution of cooperation by multilevel selection. Proceedings of the National Academy of Sciences, 103, 10952-5.

Traulsen, A., Semmann, D., Sommerfeld, R. D., Krambeck, H.-J., \& Milinski, M. (2010). Human strategy updating in evolutionary games. Proceedings of the National Academy of Sciences, 107, 2962-2966.

Triandis, H. C. (1972). The analysis of subjective culture. New York: Wiley.

Vancouver, J. B., Putka, D. J., \& Scherbaum, C. A. (2005). Testing a computational model of the goal-level effect: An example of a neglected methodology. Organizational Research Methods, 8, 100-127.

Vancouver, J. B., Tamanini, K. B., \& Yoder, R. J. (2010a). Using dynamic computational models to reconnect theory and research: Socialization by the proactive newcomer as example. Journal of Management, 36, 764-793.

Vancouver, J. B., Weinhardt, J. M., \& Schmidt, A. M. (2010b). A formal, computational theory of multiple-goal pursuit: integrating goal-choice and goal-striving processes. Journal of Applied Psychology, 95(6), 985. 
Veenhoven, R. (1989). National wealth and individual happiness (pp. 9-32). Springer Netherlands.

Wang, C. S., \& Leung, A. K. Y. (2010). The cultural dynamics of rewarding honesty and punishing deception. Personality and Social Psychology Bulletin, 0146167210385921.

Wang, C. S., Leung, A. K. Y., See, Y. H. M., \& Gao, X. Y. (2011). The effects of culture and friendship on rewarding honesty and punishing deception. Journal of Experimental Social Psychology, 47, 1295-1299.

Weibull, J. W. (1997). Evolutionary game theory. MIT press.

Winsberg, E. (2003). Simulated experiments: Methodology for a virtual world. Philosophy of science, $70,105-125$.

Ye, H., Tan, F., Ding, M., Jia, Y., \& Chen, Y. (2011). Sympathy and punishment: evolution of cooperation in public goods game. Journal of Artificial Societies and Social Simulation, 14(4), 20. 


\section{Appendix}

Derivation of Opportunistic Agent's Decision Criteria in the PGG

Opportunistic agents in our evolutionary game model cooperate or defect depending on which action is more beneficial to them, given the punishment strategies of their co-players. This decision turns out to be to cooperate if the ratio of the contribution cost $c$ over the punishment fine $\rho$ is less than the difference between the number of Responsible Punishers (R) and the number of Anti-social Punishers (A) in the neighborhood/group. This condition is derived as follows: Letting $E\left(\pi_{C}\right)$ be the expected payoff to an agent that cooperates, we have

$$
E\left(\pi_{C}\right)=E(\text { contrib }) \cdot \frac{r}{\text { groupsize }}-c-\rho \cdot[N(A)+N(S)],
$$

where $E$ (contrib) is the expected total amount of contributions of all co-players (including the agent itself) and groupsize is the size of the group to which the total public good will be distributed. The cost of contributing is $c, N(A)$ and $N(S)$ are the number of co-players in the group that punish Anti-socially and Spitefully respectively, and $\rho$ is the amount by which each punishment action would reduce the agent's payoff. Similarly, if the agent that defects, we have

$$
E\left(\pi_{D}\right)=E(\text { contrib }) \cdot \frac{r}{\text { groupsize }}-\rho \cdot[N(R)+N(S)]
$$

This agent does not incur the cost of contributing, so in contrast to Eq. 1, $c$ is not subtracted. Since the agent defects, it is punished by all the Responsible and Spiteful punishers in the group, $N(R)$ and $N(S)$. Thus, to satisfy $E\left(\pi_{C}\right)>E\left(\pi_{D}\right)$, substituting Eqns. 1 and 2, we require:

$$
\begin{gathered}
E(\text { contrib }) \cdot \frac{r}{\text { groupsize }}-c-\rho \cdot[N(A)+N(S)] \\
>E(\text { contrib }) \cdot \frac{r}{\text { groupsize }}-\rho \cdot[N(R)+N(S)] \\
\Rightarrow-c-\rho \cdot[N(A)+N(S)]>-\rho \cdot[N(R)+N(S)] \\
\Rightarrow-c-\rho \cdot N(A)>-\rho \cdot N(R)
\end{gathered}
$$




$$
\begin{aligned}
& \Rightarrow \quad-\frac{c}{\rho}-N(A)>-N(R) \\
& \Rightarrow c / \rho<N(R)-N(A)
\end{aligned}
$$

Hence, it is beneficial to cooperate, i.e. $E\left(\pi_{C}\right)>E\left(\pi_{D}\right)$, if $c / \rho<N(R)-N(A)$, which gives the condition listed above. Again, $N(R)$ and $N(A)$ are simply the number of Responsible Punishers and the number of Anti-Social Punishers in the neighborhood (or in the group playing the game), respectively. 


\section{Figure Captions}

Fig. 1. Evolutionary game theory framework for studying evolution of behaviors.

Fig. 2. Long-term average proportions of cooperation and punishment strategies under different levels of threat. The left panel shows (C)ooperators, (D)efectors, and (O)pportunistic agents. It also includes the cooperation rate, the percentage of actions in the game that are cooperation $(\%$ Cooperation). The right panel shows (R)esponsible (A)ntisocial, (S)piteful, and (N)on-punishing agents. To attain long-term average proportions, we ran simulations for 5000 timesteps and averaged the population proportions over all timesteps. Each data point in the plot is the average of 50 simulation runs. Error bars show standard deviation. Model parameters are $\mathrm{r}=3, \mathrm{c}=1, \lambda=$ $1 / 2, \rho=3 / 2, \mu=0.1, d=0.1$, base-pay $=30$. Note higher Responsible Punishers $(\mathrm{R})$ and higher cooperation by Opportunistic (O) and Cooperator (C) agents under higher threat levels.

Fig. 3. Snap shot of the population grid at different times under conditions of high threat. Left panel shows the contribution strategies and the right panel shows the punishment strategies.

Fig. 4. Changes in cooperation and punishment strategy proportions over time in a population facing a sudden threat increase or decrease at time 1000. The top panels shows (C)ooperators, (D)efectors, and (O)pportunistic agents, and the percentage of cooperation actions (\%C). The right panel shows (R)esponsible (A)ntisocial, (S)piteful, and (N)on-punishing agents. Panel a) shows a population existing in a low-threat $(\tau=5)$ environment and then the threat increases (to $\tau$ $=25)$. The population in panel $\mathrm{b})$ exists in a high-threat $(\tau=25)$ environment and then the threat 
decreases (to $\tau=5$ ). Note increases in (R)esponsible Punishers and cooperation by (O)pportunistic and (C)ooperator agents when threat is increased, and corresponding decreases when threat is decreased.

Fig. 5. Payoff matrix of our coordination game.

Fig. 6. Long-term average percentage of coordination and the population proportion of punishment strategies under different levels of threat. The plot shows Norm-(E)nforcing, (S)piteful, and (N)on-punishing agents. To attain long-term average proportions, we ran simulations for 5000 timesteps and averaged the quantities over all timesteps. Each data point in the plot is the average of 50 simulation runs. Error bars show standard deviation. Model parameters are $\lambda=1 / 2, \rho=3 / 2, \mu=0.1, d=0.1$, base-pay $=30$. Note higher Norm- $(\mathbf{E})$ nforcing Punishers and higher coordination under higher threat levels.

Fig. 7. Payoffs and fitness under high threat $(\tau=32)$ and low threat $(\tau=10)$ conditions. Circles show payoffs a hypothetical agent acquires after playing four other agents that coordinate with it, receiving a payoff of 8 plus the base payoff, squares show expected payoffs when the other agents play randomly, a payoff of 4 plus the base payoff. Figure assumes base payoff of 30 . 
Table 1. Model Parameters

\begin{tabular}{ccl}
\hline Variable & Default Value & \multicolumn{1}{c}{ Description } \\
\hline $\mathbf{r}$ & 3 & $\begin{array}{l}\text { The multiplication factor of the PGG. The sum of all } \\
\text { contributions is multiplied by it before sharing the result } \\
\text { evenly. }\end{array}$ \\
\hline $\mathbf{c}$ & 1 & The contribution/cost of contributing to agent. \\
\hline $\boldsymbol{\lambda}$ & $3 / 2$ & The punishment fine applied to the punished agent. \\
\hline $\boldsymbol{\mu}$ & $1 / 2$ & The punishment cost to the punishing agent. \\
\hline $\boldsymbol{d}$ & 0.1 & $\begin{array}{l}\text { The exploration (mutation) rate. With this probability, an } \\
\text { agent chooses a random new strategy at each time step. }\end{array}$ \\
\hline & 0.1 & $\begin{array}{l}\text { The death rate. Each agent is removed from the } \\
\text { population with this probability at each time step. }\end{array}$ \\
\hline base-pay & {$[0,5,10, \ldots]$} & $\begin{array}{l}\text { The degree of societal threat a population is exposed to. } \\
\text { The parameter is varied in increments of } 5 \text { from } 0 \text { up to } \\
\text { the amount beyond which the population seizes to be } \\
\text { able to exist (i.e., catastrophic threat, resulting in an } \\
\text { empty grid). }\end{array}$ \\
\hline & $\begin{array}{l}\text { The base payoff that agents receive regardless of any } \\
\text { interactions. }\end{array}$ \\
\hline
\end{tabular}


Table 2. Example PGG Interactions and Outcomes

\section{Interaction Group Actions/Outcome}

OR, ON, ON, CN All agents contribute. The one Responsible Punisher (R) leads the other Opportunistic agents to prefer contributing. No one punishes or is punished.

OR, OA, OA, CN None of the Opportunistic (O) agents contribute. The one Responsible Punisher $(R)$ is not enough to induce the agents to contribute, because there are two Anti-Social Punishers (A) that would punish contributing agents. The $\mathrm{R}$ agent punishes the two other $\mathrm{O}$ agents not contributing. The Cooperative (C) agent is punished by the two A agents.

ON, DN, CS, CR All agents contribute except for the Defecting (D) agent. The one Responsible Punisher (R) leads the other Opportunistic agents to prefer contributing. The $\mathrm{R}$ agent punishes the $\mathrm{D}$ agent. The one Spiteful Punishers (S) punishes all others, but doesn't influence the $\mathrm{O}$ agent to defect because he would punish contribution as will.

OR, OS, OS, ON All agents contribute, due to the Responsible Punisher (R). The Spiteful Punishers (S) punish all other agents, but since they are known to punish any action they do not deter agents from cooperating.

CR, OA, DA, OR None of the agents contribute except or the one Cooperative (C) agent. The two responsibly punishing agents $(\mathrm{R})$ are not enough to induce Opportunistic $(\mathrm{O})$ agents to contribute because there are also two Anti-Social Punishing (A) agents. The A agents punish the $\mathrm{C}$ agent. The two $\mathrm{R}$ agents punish the other two $\mathrm{O}$ agents. 


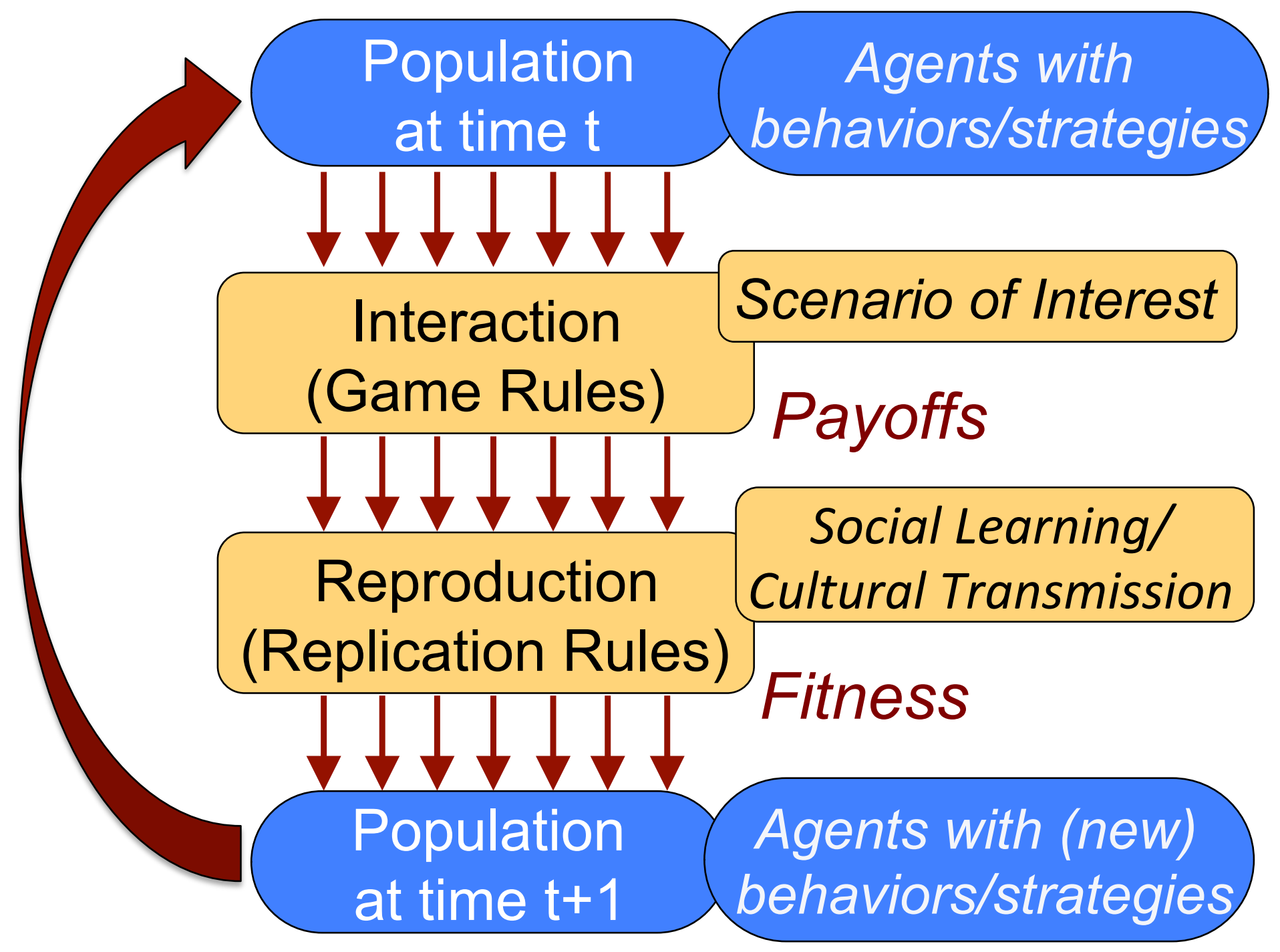




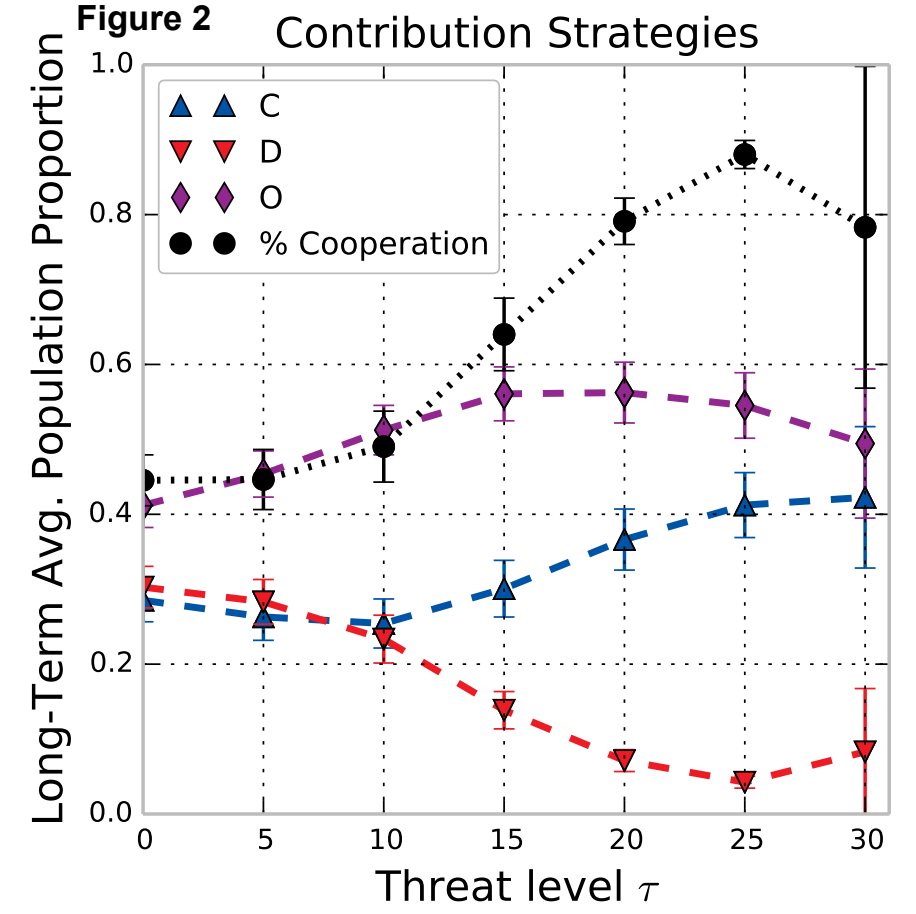

\section{Punishment Strategies}

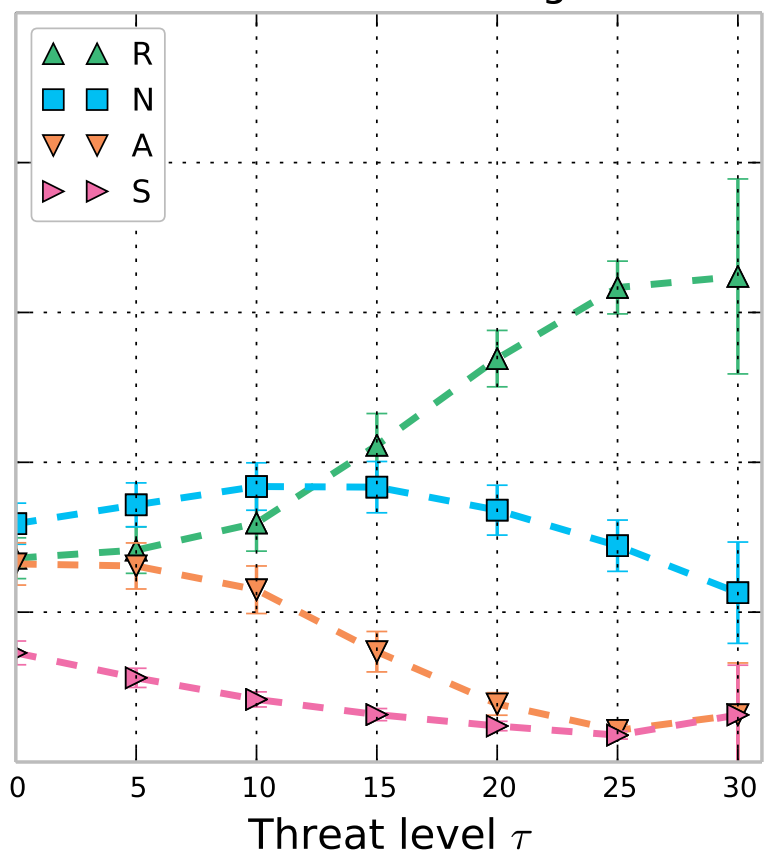


Figure 3

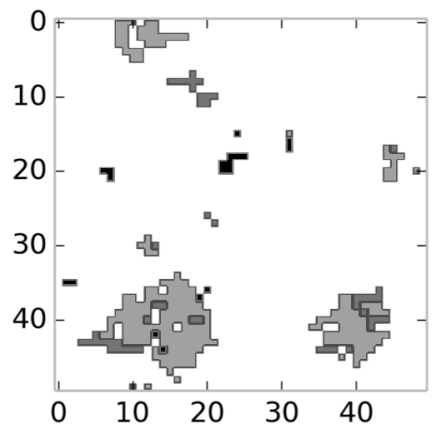

Time 20

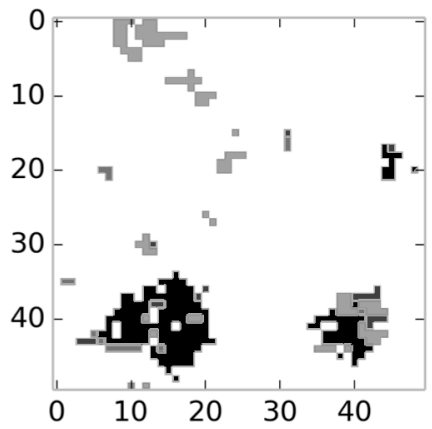

Time 40
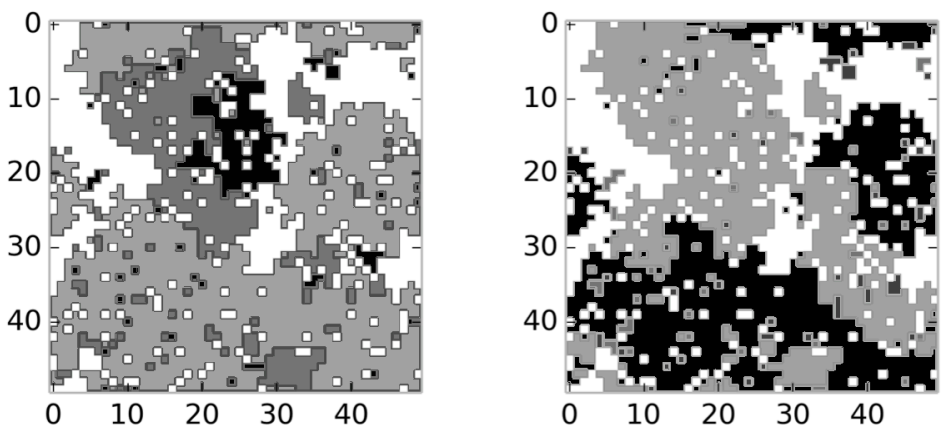

Time 300
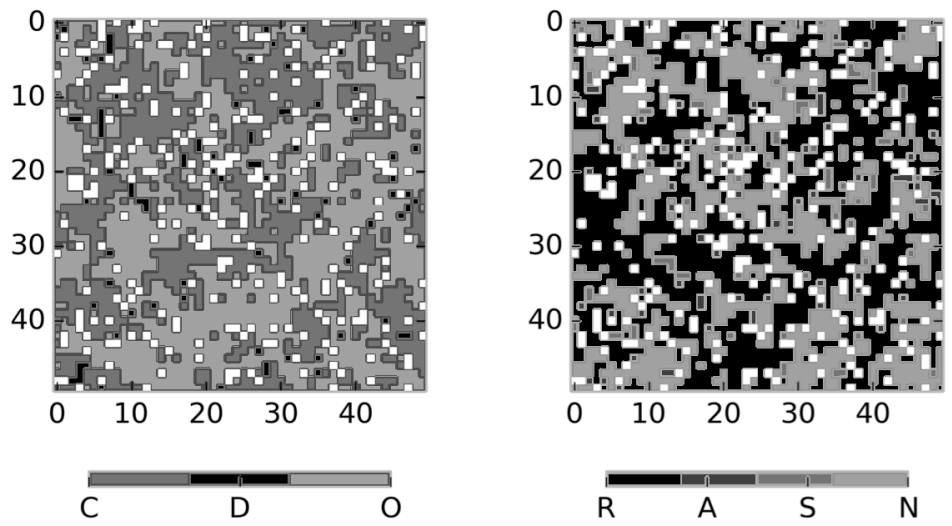

Contribution Type 
Figure 4

a) Threat Increase
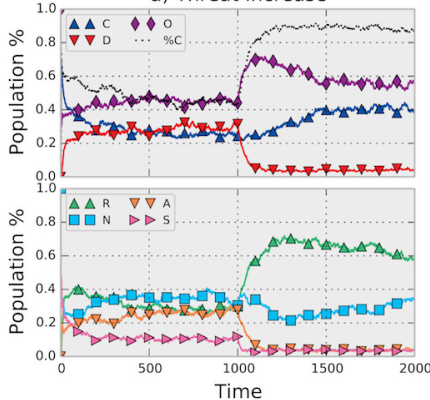

b) Threat Decrease
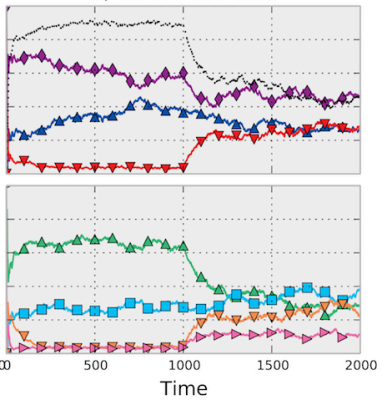
Figure 5

A

B

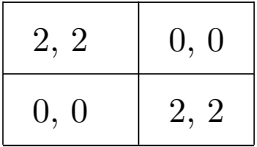


Figure 7

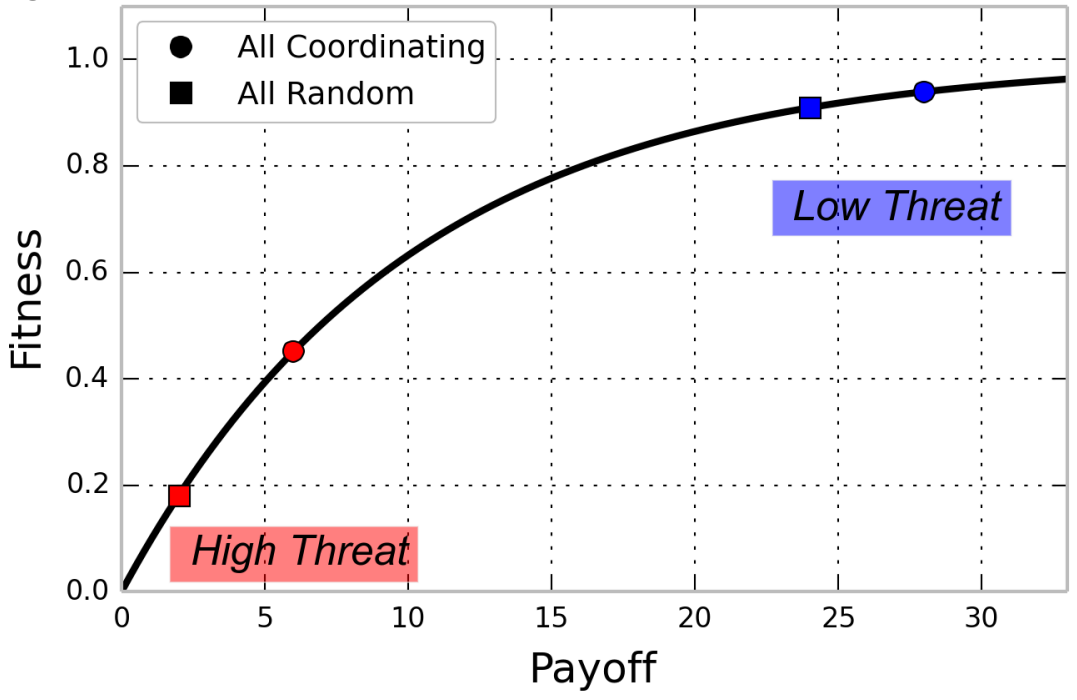

